\title{
Pituitary Adenylate Cyclase Activating Peptide Phase Shifts Circadian Rhythms in a Manner Similar to Light
}

\author{
Mary E. Harrington, ${ }^{1}$ Sabina Hoque, ${ }^{1}$ Adam Hall,, ${ }^{1,2}$ Diego Golombek, ${ }^{3}$ and Stephany Biello ${ }^{4}$ \\ ${ }^{1}$ Departments of Psychology and Biological Sciences, Smith College, Northampton, Massachusetts 01063, ${ }^{2}$ Department \\ of Biology, Mount Holyoke College, South Hadley, Massachusetts, ${ }^{3}$ Departamento de Fisiología, Facultad de Medicina, \\ Universidad de Buenos Aires, Paraguay 2155, (1121) Buenos Aires, Argentina, and ${ }^{4}$ Psychology Department, University \\ of Glasgow, Glasgow, United Kingdom G128QB
}

\begin{abstract}
The endogenous circadian pacemaker in mammals is located in the suprachiasmatic nuclei (SCN) of the hypothalamus. Various cues can reset circadian rhythm phase, thereby entraining the internal rhythm to the environmental cycle, and these effects can be investigated using an in vitro method to measure phase shifts of the SCN. Although pituitary adenylate cyclase activating peptide (PACAP) is localized in retinal inputs to the SCN, it has been reported to alter clock phase only during the subjective day (Hannibal et al., 1997), whereas light alters phase only in the subjective night. In this study we show that PACAP can reset the clock in the photic pattern during the subjective night
\end{abstract}

The suprachiasmatic nuclei (SCN) is an endogenous circadian pacemaker in mammals (Klein et al., 1991). Under constant conditions, an animal displays a free-running rhythm of $\sim 24 \mathrm{hr}$, as determined by its internal clock. Various external cues can reset circadian rhythm phase, thereby entraining the internal rhythm to the environmental cycle. Light causes a phase response pattern mimicked by various chemical and electrical treatments, such as glutamatergic agents and optic nerve electrical stimulation (Ebling, 1996; Kornhauser et al., 1996; Meijer et al., 1996; Moore, 1997). Another pattern of phase shifting involves advances during the subjective day and small delays or no shifts during the subjective night. Inputs that shift in this manner include access to a novel running wheel, application of neuropeptide $\mathrm{Y}$ or serotonergic agonists, and cAMP activators (Mrosovsky, 1995; Miller et al., 1996).

Photic information is relayed to the SCN via the retinohypothalamic tract (Ebling, 1996; Kornhauser et al., 1996; Meijer et al., 1996; Moore, 1997). Neurochemicals associated with the retinohypothalamic tract include glutamate acting at both NMDA and non-NMDA receptors, and the peptides substance $\mathrm{P}$ and pituitary adenylate cyclase activating peptide (PACAP) (Ebling, 1996; Hannibal et al., 1997, 1998; Moore, 1997).

A member of the vasoactive intestinal polypeptide (VIP)/ glucagon/secretin/growth-hormone-releasing hormone super-

\footnotetext{
Received April 16, 1999; accepted May 13, 1999.

This work was supported by National Institutes of Health grants to M.E.H., Consejo Nacional de Investigaciones Científicas y Técnicas, Agencia Nacional de Promoción Cientifica y Técnica, Universidad de Buenos Aires and Fundación Antorchas (Argentina) to D.A.G., and Royal Society grant to S.M.B. We thank Liz Cate, Jenny Siegel, and Katie Schak for technical assistance and Victoria Flood and Donna Ewell for animal care.

Correspondence should be addressed to Mary E. Harrington, Department of Psychology, Smith College, Northampton, MA 01063.

Copyright (C) 1999 Society for Neuroscience $0270-6474 / 99 / 196637-06 \$ 05.00 / 0$
}

when applied in $10 \mathrm{pm}$ to $1 \mathrm{~nm}$ doses. This appears to be mediated via a glutamatergic mechanism, possibly by potentiation of NMDA currents as is seen at 10-100 pM. Given at higher doses (>10 nm), PACAP shifts in the subjective day, apparently via activation of adenylate cyclase and increased intracellular cAMP. These results indicate dose and phase specificity of the effects of PACAP, and a new role as a transmitter in the retinohypothalamic tract.

Key words: cAMP; circadian; diurnal; glutamate; NMDA; PACAP; suprachiasmatic

family, PACAP can either activate cAMP signal cascades via stimulation of adenylate cyclase or alter inositol phosphate levels via phospholipase C (Masuo et al., 1993; Spengler et al., 1993; Arimura and Said, 1996). Both forms of PACAP (PACAP-38 and PACAP-27) are widely distributed (Masuo et al., 1993; Arimura et al., 1996). PACAP regulates hormone release from the pituitary, pancreas, and adrenal gland, and may be involved in spermatogenesis and neuritogenesis (Arimura et al., 1996). Two subclasses of PACAP receptors exist in the SCN, type I and type II (Hannibal et al., 1997). The type I receptor has high affinity for PACAP and low affinity for VIP, whereas the type II receptor has similar affinities for PACAP and VIP (Spengler et al., 1993; Arimura et al., 1996).

Although the presence of PACAP in the retinohypothalamic tract suggests a role in conveying photic information to the circadian pacemaker, the action of PACAP to increase cAMP suggests a role in mediating nonphotic phase shifts during the subjective day. One study using the in vitro rat SCN preparation demonstrated that PACAP advances the phase of the firing rate rhythm during the subjective day, an effect that can be blocked by previous treatment with the cAMP antagonist Rp-cAMPs (Hannibal et al., 1997). Similarly, we found that PACAP can phase advance the hamster in vitro SCN firing rate rhythm when applied in the subjective day (Harrington and Hoque, 1997). On the other hand, a recent report indicates that PACAP induces increases in $\left[\mathrm{Ca}^{2+}\right]_{\mathrm{i}}$ independent of cAMP (Kopp et al., 1999). Here we report results of further studies using the hamster brain slice preparation to measure phase shifts induced by PACAP.

\section{MATERIALS AND METHODS}

Brain slice studies. Male golden hamsters ( $>1$ mo of age; Charles River Laboratories, Kingston, NY) were housed under a 14/10 light/dark cycle, and animal care was in accordance with Smith College institutional guidelines. Brain slices were prepared between Zeitgeber time (ZT) 
0-10, with ZT 12 arbitrarily defined as the time of lights off. Hamsters were administered an overdose of halothane, and the brain was quickly dissected. A mechanical tissue chopper was used to prepare coronal hypothalamic slices $(500-\mu \mathrm{m}$-thick). Slices were placed into a gas:fluid interface chamber. Tissue was maintained at the interface between artificial CSF (ACSF) and warm, humidified $95 \% \mathrm{O}_{2}$ and $5 \% \mathrm{CO}_{2}$. ACSF containing (mM): $125.2 \mathrm{NaCl}, 3.8 \mathrm{KCl}, 1.2 \mathrm{KH}_{2} \mathrm{PO}_{4}, 1.8 \mathrm{CaCl}_{2}$, $1.0 \mathrm{MgSO}_{4}, 24.8 \mathrm{NaHCO}_{3}$, and 10 glucose, $\mathrm{pH} 7.4$, was supplemented with an antibiotic (gentamicin, $50 \mathrm{mg} / \mathrm{l}$ ) and a fungicide (amphotericin, $2 \mathrm{mg} / \mathrm{l}$ ) and maintained at $34.5^{\circ} \mathrm{C}$. In some cases we simultaneously recorded from two SCN slices taken from one hamster. In these cases, the two slices were given different drug applications, so that the data in any one experimental group always represents results from slices from different individuals. Both PACAP-38 and Rp-cAMPS (adenosine 3', $5^{\prime}$ cyclic monophosphothioate, Rp-isomer) were supplied by Sigma (St. Louis, MO). AP-5 [( \pm )-2-amino-5-phosphonopentanoic acid] was purchased from Research Biochemicals International (Natick, MA). All drugs were applied as a $200 \mathrm{nl}$ microdrop to the SCN on the first day in vitro. Extracellular single-unit activity in the SCN was measured using glass micropipettes filled with ACSF advanced through the slice with a hydraulic microdrive. The signal was amplified, filtered, and discriminated, and firing rate was measured using a rate monitor and computer. The spontaneous firing rate of each cell was measured for $1 \mathrm{~min}$. The average firing rate of each cell was plotted versus the $\mathrm{ZT}$ of recording. Recordings from individual slices were generally conducted for 6-7 hr per day on the second day in vitro. Data were grouped into $1 \mathrm{hr}$ bins and analyzed by an ANOVA. If the ANOVA indicated significant differences $(p<0.05)$ across the bins, data were summarized using a $1 \mathrm{hr}$ running mean smoother with a $15 \mathrm{~min}$ lag, and the time of peak firing rate calculated as the $\mathrm{ZT}$ at the middle of the $1 \mathrm{hr}$ bin with the highest value. The person recording the firing rate data was blind to the drug treatments of the slice, except in one recording (Rp-cAMPS at ZT $6 ; n=1)$.

Patch-clamp studies. Pregnant female golden hamsters (Charles River Laboratories) and pups were housed under a 14/10 light/dark cycle with animal care in accordance with Smith College institutional guidelines. SCN neurons were acutely dissociated from brain slices of 5- to 15-d-old hamster pups using the following protocol. Between ZT 0 and 10, pups were overdosed with halothane anesthesia and decapitated. Brains were quickly dissected in ice-cold ACSF; $500 \mu \mathrm{m}$ hypothalamic slices containing the SCN were prepared and placed in ACSF bubbled with $95 \% \mathrm{O}_{2}$ and $5 \% \mathrm{CO}_{2}$ at room temperature for $1 \mathrm{hr}$. The SCNs were then carefully cut out with a razor blade and digested in ACSF containing 1.5 $\mathrm{mg} / \mathrm{ml}$ protease (type XIV from bacterial Streptomyces griseus; Sigma) at $37^{\circ} \mathrm{C}$ for $5 \mathrm{~min}$. The tissue was then washed twice in HEPES-buffered minimum essential medium (MEM; Sigma), and cells were dissociated by gentle trituration in 200-300 $\mu$ l of MEM with a fire-polished Pasteur pipette. The cell suspension was deposited in drops on poly-D-lysinetreated strips of glass coverslip, and cells were allowed to settle for $15 \mathrm{~min}$ before flooding with MEM. Cells were maintained at room temperature under a $95 \% \mathrm{O}_{2}$ and $5 \% \mathrm{CO}_{2}$ atmosphere and remained viable for recording for $4-5 \mathrm{hr}$. Coverslips were positioned in the recording bath, and small SCN neurons of 5-10 $\mu \mathrm{m}$ diameter (unipolar, bipolar, and multipolar) were easily identified by their morphology. Cells were superfused with (in mM): $140 \mathrm{NaCl}, 2.5 \mathrm{KCl}, 3 \mathrm{CaCl}_{2}, 10 \mathrm{HEPES}, 3$ glucose, and $0.1 \mu \mathrm{M}$ tetrodotoxin, $\mathrm{pH} 7.4$ with $\mathrm{NaOH}$. Single-channel currents were recorded from outside-out membrane patches using standard techniques. Fire-polished, borosilicate glass pipettes (World Precision Instruments), fabricated by a two-stage pull (PB-7 puller; Narishige, Tokyo, Japan) and filled with a solution containing (in mM): $120 \mathrm{~K}$ gluconate, 20 $\mathrm{KCl}, 5 \mathrm{NaCl}, 2 \mathrm{MgCl}_{2}, 1 \mathrm{CaCl}_{2}, 1.1 \mathrm{EGTA}, 1 \mathrm{ATP}\left(\mathrm{Na}^{+}{ }^{+}\right.$salt), 1 GTP $\left(\mathrm{Na}^{+}\right.$salt), and 10 HEPES, $\mathrm{pH} 7.3$ with $\mathrm{KOH}$, typically had resistances of 5-10 M $\Omega$. Membrane patches were held at -50 to $-60 \mathrm{mV}$ and were exposed to combinations of $20 \mu \mathrm{M}$ NMDA, $100 \mathrm{~nm}$ glycine, and varying PACAP concentrations by bath (volume, 200-300 $\mu \mathrm{l}$ ) exchange (flow rate, $3 \mathrm{ml} / \mathrm{min}$ ). All currents were acquired using an Axoclamp 200A interfaced to a computer running pClamp 6.1 software (Axon Instruments, Foster City, CA). Data were filtered at $1 \mathrm{kHz}$ with a low-pass 8-pole Bessel filter, sampled at $4 \mathrm{kHz}$ and analyzed using pClamp 6.1. The duration of openings for NMDA channels at all integral current levels was assessed throughout the 1 min exposure, a percentage of time spent in opening was calculated, and values for exposures with and without PACAP were compared.

In vivo experiments. Male Syrian hamsters, 40- to 60-d-old, were purchased from Charles River (Margate, Kent, UK) and held in LD14: 10. Between 11 and $12 \mathrm{~d}$ after arrival, animals were anesthetized with sodium pentobarbital ( $\sim 80 \mathrm{mg} / \mathrm{kg}$, i.p.) and fitted with a 25 gauge stainless steel guide cannula aimed at the SCN. The cannulae were stereotaxically placed so that the tip was $2.7 \mathrm{~mm}$ dorsal to the SCN and entered the brain slightly off midline at a $10^{\circ}$ angle to avoid damaging the midsagittal sinus. Cannulae were held in place with dental cement, and three small screws were anchored in the skull. A pin consisting of a 10 $\mathrm{mm}$ length of 30 gauge stainless steel wire was inserted into the guide cannula to prevent blockage. After $14-15 \mathrm{~d}$ of recovery, hamsters were placed in constant dim red light (mean, 24 lux) provided by safelight lamps fitted with Kodak (Eastman Kodak, Rochester, NY) filter OA 152-1491. Pilot tests indicated that exposure to this safe light at circadian time (CT) $1,5,9,13,17$, and 21 for up to $15 \mathrm{~min}$ had no effect on the free-running rhythm of hamsters held in constant darkness (Biello, 1995). The onset of wheel-running, designated as CT 12, was used as the phase reference point when timing cannula injections. The onset was defined as 100 wheel revolutions followed by 100 wheel revolutions within the next 30 min. Phase shifts were determined by calculating a regression line for the five onsets before the manipulation and extrapolated to the day of the pulse. The activity onset on the pulse day as well as the following two onsets were omitted from the regression calculation. A second regression line was calculated from the third through seventh postpulse onsets and projected back to the pulse day. The difference between the onsets projected from the prepulse and from the postpulse regression lines was taken as the phase shift. Microinjections were performed under a red safe light fitted with a Kodak OA 152-1491 filter. Pilot tests indicated that exposure to this light at CT 14 for up to $15 \mathrm{~min}$ had no effect on the free-running rhythm of hamsters held in constant darkness $(n=5 ; \mathrm{S}$. Biello, unpublished data). PACAP or ACSF were injected through a $1 \mu \mathrm{l}$ Hamilton syringe connected by $\sim 10 \mathrm{~cm}$ of polyethylene tubing to a 13.1 $\mathrm{mm}$ stainless steel injection cannula (30 gauge). During the injections the animals were restrained by hand. After the pin was removed from the guide cannula, the injection cannula was inserted, the plunger was depressed over a $30 \mathrm{sec}$ period, and the injector was held in place for a further $30 \mathrm{sec}$. Solutions were freshly prepared on the day of treatment and delivered in a volume of $200 \mathrm{nl}$. Hamsters were allowed at least $10 \mathrm{~d}$ between treatments. At the conclusion of the experiment, injections sites were verified by an injection of $200 \mathrm{nl}$ of ink. Hamsters were perfused, and $40 \mu \mathrm{m}$ sections were cut using a cryostat. Data were only included from hamsters if the cannula tip was within $400 \mu \mathrm{m}$ of the SCN, the SCN showed no apparent gliosis, ink was found to enter the SCN, and no ink was found in the ventricular system.

\section{RESULTS}

Hamster SCN cells express a reliable increase in spontaneous firing rate for $\sim 1 \mathrm{hr}$ in the midsubjective day (ZT 6.3) in control experiments. The dose-response relationship for phase shifts in the time of peak firing after applications of PACAP at ZT 6 is shown in Figure $1 A$. Supporting previous reports (Hannibal et al., 1997; Harrington and Hoque, 1997), we found phase advance shifts after treatment with doses of $10 \mathrm{~nm}$ to $1 \mu \mathrm{M}$. A $1 \mu \mathrm{M}$ dose at ZT 14 or ZT 18 did not significantly phase shift the rhythm (Fig. $1 C$ ). Thus, a $1 \mu \mathrm{M}$ dose of PACAP appears to induce phase advance shifts in the subjective day and no phase shifts in the subjective night.

We reexamined the phase-shifting effects of PACAP during the subjective night. We replicated previous results showing that a 1 $\mu \mathrm{M}$ dose of PACAP at ZT 14 did not phase shift the rhythm (Hannibal et al., 1997). However, lower doses of PACAP were able to induce phase delay shifts at ZT 14, early in the subjective night (Fig. $1 B$ ). The dose-response relationship for PACAP at ZT 14 indicated maximal phase delays after applications of $1 \mathrm{~nm}$ PACAP. This dose of PACAP also induced a phase advance when applied at ZT 18 on the first day in vitro, but had no significant phase-shifting effect at ZT 6 (Fig. 1C). Therefore, a 1 nM dose of PACAP appears to induce phase shifts in the photic pattern.

Because previous studies indicated that PACAP can potentiate NMDA currents (Liu and Madsen, 1997, 1998; Wu and Dun, 1997), we tested SCN neurons for a similar effect. Outside-out 
A.



B.

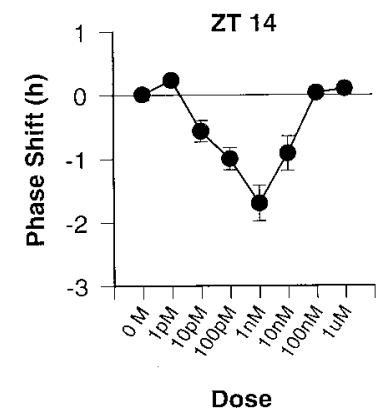

Figure 1. Dose- and phase-dependent effects of PACAP. PACAP was applied on the first day in vitro on the phase of the rhythm in firing rate recorded from SCN neurons on the second day. $A$, Dose-response curve for PACAP applied at ZT 6, midsubjective day. $B$, Dose-response curve for PACAP applied at ZT 14. Phase shifts are measured relative to control slices, untreated, or given microdrop applications of ACSF at either ZT 6, 14, or 18, which showed peak firing at ZT $6.3( \pm 0.1, n=7$; range, 6.0-6.7). Each symbol represents the mean \pm SEM phase shift of $n=3$ slices. $C$, Comparison of the phase-shifting responses to two doses of PACAP (1 nM vs $1 \mu \mathrm{M})$ at three phases: ZT 6, ZT 14, and ZT 18. Means \pm SEMs are shown; $n=3$ in all groups.

patches were excised from acutely isolated SCN neurons and held at membrane potentials of -50 to $-60 \mathrm{mV}$. After exposure to 20 $\mu \mathrm{M}$ NMDA-100 $\mathrm{nm}$ glycine in the recording medium, channel activity was observed in all patches. NMDA-induced activity typically had conductance of $40-50 \mathrm{pS}$ and there were few contaminating currents. Exposures of 1 min duration every $2 \mathrm{~min}$ gave consistent levels of activity, and responses were nondesensitizing throughout the duration of the application. Inclusion of PACAP (10 pM) in the NMDA-glycine solution consistently resulted in an enhanced level of channel activity throughout the exposure (Fig. 2A). The potentiating effects of PACAP were always fully reversible, with channel activity returning to control levels after subsequent exposure to NMDA-glycine alone. The effects of PACAP on NMDA channel activity were dosedependent (Fig. 2B). At low concentrations (1-100 pM), the inclusion of PACAP predominantly resulted in potentiated levels of channel activity. However, at concentrations above $1 \mathrm{~nm}$, PACAP either had no effect or significantly inhibited activity levels (Fig. 2B). Inhibitory effects of PACAP were also reversible. By using excised membrane patches, we demonstrate that PACAP can potentiate NMDA currents in SCN neurons independent of cytoplasmic factors.

To test the hypothesis that PACAP phase delays the clock at ZT 14 via a glutamatergic mechanism, possibly involving potentiation of NMDA currents, we applied the NMDA receptor

A

a)

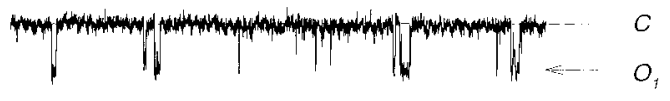

b)



c)

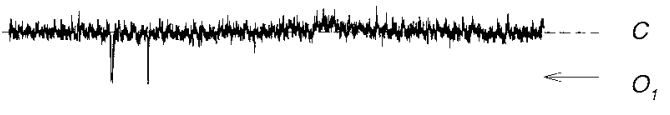

B

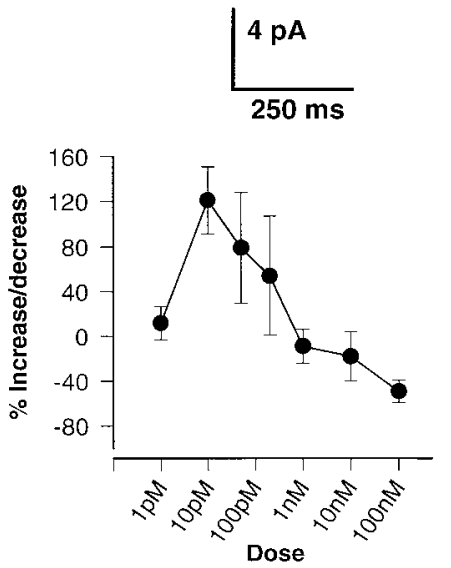

Figure 2. A, PACAP has a dual, potentiating, and inhibitory effect on NMDA-induced channel activity in outside-out patch recordings from SCN neurons. A patch was held at $-60 \mathrm{mV}$ and exposed to $20 \mu \mathrm{M}$ NMDA-100 nM glycine in the absence and presence of two concentrations of PACAP. $a$, Sample trace of activity in the presence of NMDAglycine alone. $b$, Trace during exposure to NMDA-glycine and $10 \mathrm{pM}$ PACAP demonstrating increased activity. $c$, Trace during exposure of the same patch to NMDA-glycine and $100 \mathrm{~nm}$ PACAP showing the pronounced reduction in $P_{\text {open }}$. $B$, Dose-dependent effects of PACAP on NMDA channel activity. Outside-out patches, held at -50 to $-60 \mathrm{mV}$, were exposed to $20 \mu \mathrm{M}$ NMDA-100 nM glycine. Values for the proportion of time spent in open configurations were assessed for channels in the absence and presence of varying concentrations of PACAP, and potentiations and inhibitions were calculated as percentages above and below control activity levels. Symbols indicate means, and error bars indicate SEMs. Each point is averaged from at least five outside-out patch recordings.

antagonists MK-801 (50 $\mu \mathrm{M})$ or AP-5 (100 $\mu \mathrm{M}$ or $1 \mathrm{mM})$ before PACAP. Both NMDA receptor antagonists were able to block PACAP phase delay shifts (1 nM PACAP at ZT 14), and had no effect when applied alone (Fig. 3B). The AMPA-kainate receptor antagonist CNQX $(10 \mu \mathrm{M})$ had no effect on the PACAP phase delay (1 nM PACAP at ZT 14) and had no phase-shifting effect when applied alone. When applied in the subjective day, MK-801 $(50 \mu \mathrm{M})$ or AP-5 (1 mM) had no effect on PACAP-induced phase advances (10 nM PACAP at ZT 6). On the other hand, treatment with the cAMP antagonist Rp-cAMPS reduced these daytime phase advances, similar to results from the previous work using rats (Hannibal et al., 1997) (Fig. 3A). Antagonizing cAMP with Rp-cAMPS had no effect on the PACAP-induced phase delays at ZT 14 (Fig. 3B). These findings support the conclusion that PACAP phase advances the clock in the subjective day by increas- 


\section{A. ZT6}

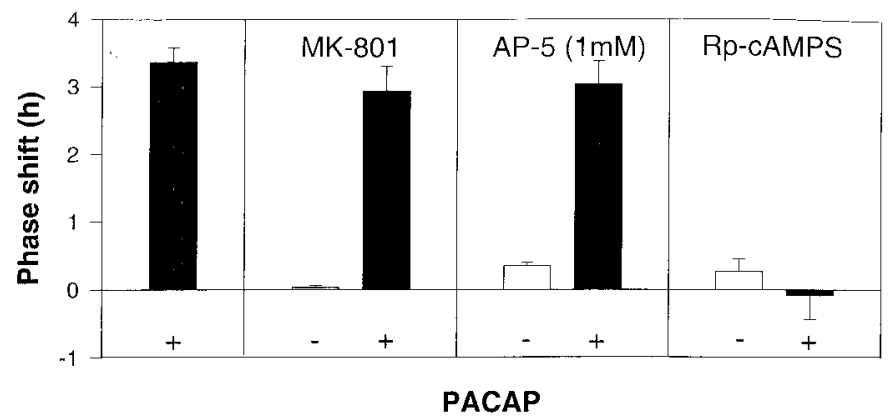

B. $\mathrm{ZT} 14$

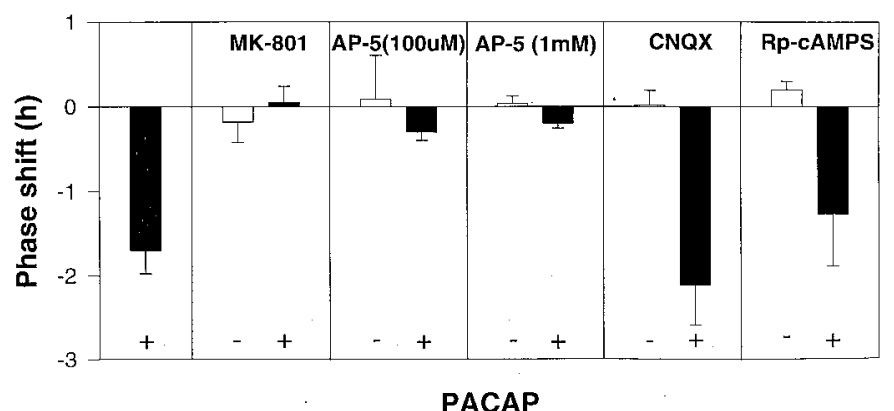

Figure 3. A, PACAP phase advances the clock in the subjective day by increasing cAMP activation. $B$, Phase delays the clock in the subjective night by a glutamatergic mechanism. SCN slices were treated with either PACAP alone or PACAP applied 5 min after application of either MK-801 $(50 \mu \mathrm{M})$, AP-5 (100 $\mu \mathrm{M}$ or $1 \mathrm{mM})$, CNQX $(10 \mu \mathrm{M})$, or Rp-cAMPs $(10 \mu \mathrm{M})$. The dose of PACAP was chosen from the dose-response curves shown in Figure 1 (10 nM PACAP at ZT 6; 1 nM PACAP at ZT 14). Histograms indicate the mean \pm SEM phase shift of $n=3$ slices, except two control groups with $n=2$ : AP-5 at ZT 6 and Rp-cAMPS at ZT 14.

ing cAMP activation, and phase delays the clock in the subjective night by a glutamatergic mechanism. Thus, two different mechanisms are mediating the subjective day and subjective night phase shifts of PACAP.

Cannula microinjections of $1 \mathrm{nM}$ PACAP to the area of the SCN induced small but significant phase shifts in wheel-running behavior. This pattern of phase shifting resembled phase shifts to light with phase delays seen at CT 14-14.5 and advances observed at CT 18-18.5 (Fig. 4). The magnitude of these shifts was less than those seen in the in vitro slice preparation.

\section{DISCUSSION}

The doses associated with a photic effect of PACAP in this study are in agreement with previous reports. PACAP is an exceptionally potent peptide. PACAP can increase $\left[\mathrm{Ca}^{2+}\right]_{\mathrm{i}}$ and stimulate insulin release from rat pancreatic islets in doses as low as $10^{-14}$ to $10^{-13} \mathrm{M}$ (Yada et al., 1994). In doses as low as $10^{-12} \mathrm{M}$ PACAP increases $\left[\mathrm{Ca}^{2+}\right]_{i}$ in dissociated supraoptic neurons and stimulates vasopressin release from supraoptic slices (Shibuya et al., 1998). The neuroprotective effect of PACAP in cultured rat cortical neurons shows a bell-shaped dose-response curve with maximal effects in the picomolar range (Morio et al., 1996). Although we have not directly measured the amount of PACAP
A.

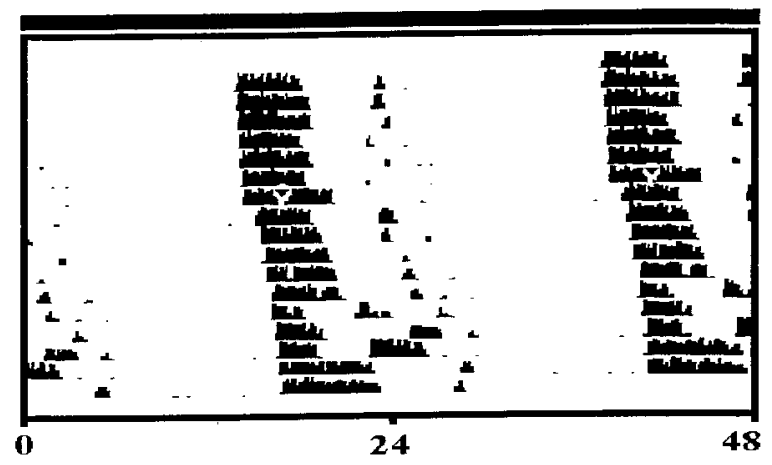

B.

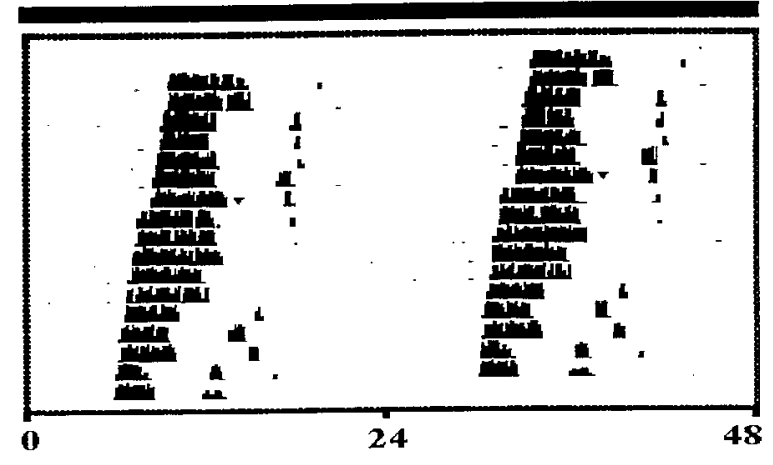

C.

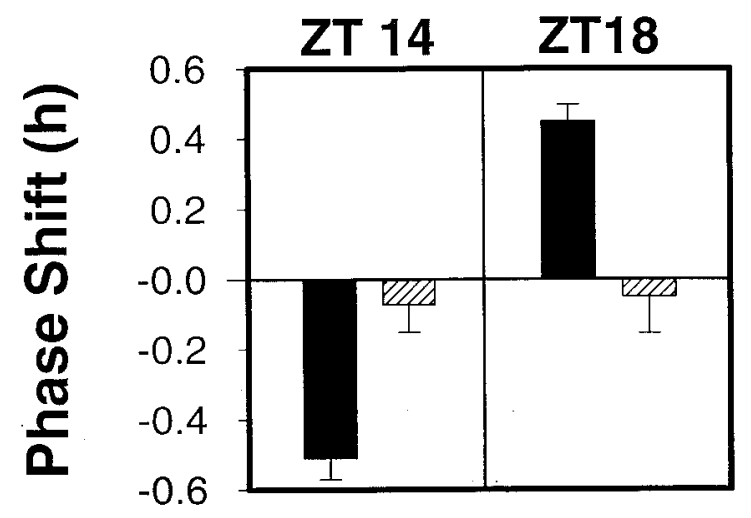

Figure 4. Administration of PACAP in vivo via a cannula aimed at the SCN resets the circadian clock in a manner similar to light. Doubleplotted actograms show daily activity of individual hamsters with the activity of each day plotted below the previous day. Arrowheads indicate PACAP administration on day 7. $A$, Sample actogram from a hamster given PACAP at CT 14. B, Sample actogram from a hamster given PACAP at CT 18. $C$, Administration of PACAP at CT 14 produced significant phase delays $(n=13$; mean phase shift, $-0.51 \pm 0.06$ SEM) when compared with ACSF controls $(n=13$; mean phase shift, $-0.07 \pm$ 0.9 SEM) on a paired $t$ test $(p<0.001)$. PACAP microinjected at CT 18 produced small but significant phase advances $(n=8$; mean, $0.45 \pm 0.39$ SEM) when compared with ACSF controls at CT $18(n=8$; mean, $-0.05 \pm 0.78 \mathrm{SEM})$ on a paired $t$ test $(p<0.005)$.

released from hamster retinal terminals after photic stimulation, it is likely that light would release the lower doses (picomolar to nanomolar range) of PACAP, because these doses altered phase in a manner similar to light. Although PACAP in the low nanomolar range $(0.5-2 \mathrm{nM})$ potentiates glycine-mediated NMDA currents in chick cortical neurons, $10-1000 \mathrm{~nm}$ concentrations of the peptide have an inhibitory effect on this current (Liu and Madsen, 1997, 1998; Wu and Dun, 1997; current study). For chick 
cortical neurons, the magnitude of the potentiating effect of PACAP on NMDA currents depends strongly on the amount of glycine present, suggesting that PACAP may interact with the glycine site to produce enhancement of NMDA currents (Liu and Madsen, 1997, 1998; Wu and Dun, 1997). Thus, the potentiation of PACAP could be regulated by the amount of coincident glycine release. Alternatively, although the potentiating effects of PACAP on NMDA currents have been recorded in excised outside-out patches (Liu and Madsen, 1997; current study), membrane-delimited modulation of NMDA currents by other mechanisms, e.g., G-proteins (Yu et al., 1997) or phosopholipase activation (Spengler et al., 1993) cannot be ruled out. Of particular interest is work indicating that PACAP potentiates glutamate-evoked release of arachidonic acid from mouse cortical neurons via a mechanism that does not appear to involve $G_{s}$ proteins, or the cAMP-PKA pathway or the phospholipase C-PKC pathway (Stella and Magistretti, 1996).

PACAP induced a similar pattern of phase shifts in vivo and in vitro in this study, whereas magnitude of the shifts varied between the two preparations. This variation could be caused by a multitude of factors, for example, the lack of feedback inhibition in the isolated SCN.

PACAP content in the rat SCN and pineal gland exhibits a significant diurnal rhythm in animals housed under light/dark cycles which disappears after housing in constant darkness, suggesting an exogenous origin of the rhythm (Fukuhara et al., 1997, 1998). These day-night variations in the SCN and in the pineal gland support a putative role of this peptide in entrainment to the daily photoperiod.

The mechanism through which PACAP is able to induce photic-like phase shifts may be related to potentiation of the effects of glutamate in the SCN. Indeed, PACAP has been shown to potentiate glutamate-induced Fos expression in cortical neurons (Martin et al., 1995). Both light and PACAP can induce phosphorylation of the transcription factor cAMP response element-binding protein (CREB) in the rat SCN (Kornhauser et al., 1996; Kopp et al., 1997), although the phase specificity of this effect of PACAP differs from that of light (von Gall et al., 1998). Phosphorylated CREB can induce immediate early gene transcription, an event associated with photic phase shifts of the circadian clock (Kornhauser et al., 1996). PACAP has also been shown to stimulate the expression of BDNF mRNA in mouse cortical neurons, an effect blocked by NMDA receptor antagonists (Pellegri et al., 1998).

These results allow insight into the actions of this peptide associated with the retinohypothalamic tract, indicating that phase shifts in the photic pattern are observed at nanomolar doses. Several more general insights are possible. First, the action of the peptide on the circadian clock differs with dose, in that lower doses (circa $1 \mathrm{~nm}$ ) phase shift in the photic pattern, apparently via potentiation of NMDA currents, whereas higher doses (circa $1 \mu \mathrm{M}$ ) phase shift in a different pattern, presumably via activation of cAMP. Second, the dose-response relationship for the photic effects of PACAP has a roughly bell-shaped curve (Figs. $1 B, 2 C$ ), suggesting that studies testing only relatively high doses of PACAP may miss this response completely (Piggins et al., 1998). Neuropeptides may vary in the amount released so as to have different cellular effects. It appears that thorough doseresponse studies conducted at several circadian phases are necessary to understand the action of a drug on a neural system (Miller, 1993).

\section{REFERENCES}

Arimura A, Said SI (1996) VIP, PACAP, and related peptides. Second International Symposium, Annals of the New York Academy of Science, Vol 805. New York: New York Academy of Science.

Biello SM (1995) Neuropeptide Y and non-photic phase shifts. University of Toronto, $\mathrm{PhD}$ thesis.

Ebling FJ (1996) The role of glutamate in the photic regulation of the suprachiasmatic nucleus. Prog Neurobiol 50:109-132.

Fukuhara C, Suzuki N, Matsumoto Y, Nakayam Y, Aoki K, Tsujimoto G, Inouye S-IT, Masuo Y (1997) Day-night variation of pituitary adenylate cyclase-activating polypeptide (PACAP) level in the rat suprachiasmatic nucleus. Neurosci Lett 229:49-52.

Fukuhara C, Inouye S-IT, Matsumoto Y, Tsujimoto G, Aoki K, Masuo Y (1998) Pituitary adenylate cyclase-activating polypeptide rhythm in the rat pineal gland. Neurosci Lett 241:115-118.

Hannibal J, Ding JM, Chen D, Fahrenkrug J, Larsen PJ, Gillette MU, Mikkelsen JD (1997) Pituitary adenylate cyclase-activating peptide (PACAP) in the retinohypothalamic tract: A potential daytime regulator of the biological clock. J Neurosci 17:2637-2644.

Hannibal J, Fahrenkrug J, Ottersen OP, Moller M (1998) PACAP and glutamate is co-stored in retinal ganglion cells and nerve terminals in the suprachiasmatic nucleus of the rat: a light- and electron microscopic study. Soc Neurosci Abstr 24:1918.

Harrington ME, Hoque S (1997) NPY opposes PACAP phase shifts via receptors different from those involved in NPY phase shifts. NeuroReport 8:2677-2680.

Klein DC, Moore RY, Reppert SM (1991) Suprachiasmatic nucleus: the mind's clock. New York: Oxford UP.

Kopp M, Meissl H, Korf HW (1997) The pituitary adenylate cyclaseactivating polypeptide-induced phosphorylation of the transcription factor CREB (cAMP response element binding protein) in the rat suprachiasmatic nucleus is inhibited by melatonin. Neurosci Lett 227:145-148.

Kopp MD, Schomerus, Dehghani F, Korf H-W, Meissl H (1999) Pituitary adenylate cyclase-activating polypeptide and melatonin in the suprachiasmatic nucleus: effects on the calcium signal transduction cascade. J Neurosci 19:206-219.

Kornhauser JM, Ginty DD, Greenberg ME, Mayo KE, Takahashi JS (1996) Light entrainment and activation of signal transduction pathways in the SCN. Prog Brain Res 111:133-145.

Liu GJ, Madsen BW (1997) PACAP38 modulates activity of NMDA receptors in cultured chick cortical neurons. J Neurophysiol 78:2231-2234.

Liu GJ, Madsen BW (1998) Modulatory action of PACAP27 on NMDA receptor channel activity in cultured chick cortical neurons. Brain Res 791:290-294.

Martin JL, Gasser D, Magistretti PJ (1995) Vasoactive intestinal peptide and pituitary adenylate cyclase-activating polypeptide potentiate c-fos expression induced by glutamate in cultured cortical neurons. J Neurochem 65:1-9.

Masuo Y, Suzuki N, Matsumoto H, Tokito F, Matsumoto Y, Tsuda M, Fujino M (1993) Regional distribution of pituitary adenylate cyclase activating polypeptide (PACAP) in the rat central nervous system as determined by sandwich-enzyme immunoassay. Brain Res 602:57-63.

Meijer JH, Watanabe K, Detari L, deVries MJ, Albus H, Treep JA, Schaap J, Rietveld WJ (1996) Light entrainment of the mammalian biological clock. Prog Brain Res 111:175-190.

Miller JD (1993) On the nature of the circadian clock in mammals. Am J Physiol 264:R821-R832.

Miller JD, Morin LP, Schwartz WJ, Moore RY (1996) New insights into the mammalian circadian clock. Sleep 19: 641-667.

Moore RY (1997) Chemical neuroanatomy of the mammalian circadian system. In: Handbook of experimental pharmacology, Vol 125, Chap 4 (Redfern PH, Lammer B, eds), pp 79-93. Berlin: Springer.

Morio H, Tatsuno I, Hirai A, Tamura Y, Saito Y (1996) Pituitary adenylate cyclase-activating polypeptide protects rat-cultured cortical neurons from glutamate-induced cytotoxicity. Brain Res 741:82-88.

Mrosovsky N (1995) A non-photic gateway to the circadian clock of hamsters. Ciba Found Symp 183:154-167.

Pellegri G, Magistretti PJ, Martin J-L (1998) VIP and PACAP potentiate the action of glutamate on BDNF expression in mouse cortical neurones. Eur J Neurosci 10:272-280.

Piggins HD, Goguen D, Rusak B (1998) Effects of microinjections of 
PACAP into the suprachiasmatic region on hamster behavioural rhythms. Soc Res Biol Rhythms Abstr 6:44.

Shibuya I, Noguchi J, Tanaka K, Harayama N, Inoue Y, Kabashima N, Ueta Y, Hattori Y, Yamashita H (1998) PACAP increases the cytosolic $\mathrm{Ca} 2+$ concentration and stimulates somatodendritic vasopressin release in rat supraoptic neurons. J Neuroendocrinol 10:31-42.

Spengler D, Waeber C, Pantaloni C, Holsboer F, Bockaert J, Seeburg PH, Journot L (1993) Differential signal transduction by five splice variants of the PACAP receptor. Nature 365:170-175.

Stella N, Magistretti PJ (1996) Vasoactive intestinal peptide (VIP) and pituitary adenylate cyclase-activating polypeptide (PACAP) potentiate the glutamate-evoked release of arachidonic acid from mouse cortical neurons. J Biol Chem 271:23705-23710.

Von Gall C, Duffield GE, Hastings MH, Kopp MDA, Dehghani F, Korf
H-W, Stehle JH (1998) CREB in the mouse SCN: a molecular interface coding the phase-adjusting stimuli light, glutamate, PACAP, and melatonin for clockwork access. J Neurosci, in press.

Wu SY, Dun NJ (1997) Potentiation of NMDA currents by pituitary adenylate cyclase activating polypeptide in neonatal rat sympathetic preganglionic neurons. J Neurophysiol 78:1175-1179.

Yada T, Sakurada M, Ihida K, Nakata M, Murata F, Arimura A, Kikuchi M (1994) Pituitary adenylate cyclase activating polypeptide is an extraordinarily potent intra-pancreatic regulator of insulin secretion from islet $\beta$-cells. J Biol Chem 269:1290-1293.

Yu SP, Sensi SL, Canzoniero LMT, Buisson A, Choi DW (1997) Membrane-delimited modulation of NMDA currents by metabotropic glutamate receptor subtypes $1 / 5$ in cultured mouse cortical neurons. J Physiol (Lond) 499:721-732. 